\title{
EDITORIAL
}

\section{Chemerin/ChemR23 pathway: a system beyond chemokines}

\author{
Florenzo lannone* and Giovanni Lapadula \\ See related research by Berg et al., http://arthritis-research.com/content/12/6/R228
}

\begin{abstract}
Chemerin is a chemokine that, through the engagement of its counter-receptor, ChemR23, attracts pro-inflammatory cells. However, chemerin has been shown to play other functions and a recent study by Berg and colleagues demonstrates that chemerin/ ChemR23 is a system beyond chemokines. Human articular chondrocytes produce chemerin and express ChemR23, and upon stimulation with recombinant chemerin increase the production of pro-catabolic cytokines and metalloproteinases. The latter are up-regulated in osteoarthritic cartilage and cause extracellular matrix breakdown. Since an increase of chemerin in fat tissue and serum of obese patients has been reported, this new feature of chemerin may represent a functional link between obesity and osteoarthritis.
\end{abstract}

Knowledge of the pathophysiology of articular cartilage is crucial in understanding the mechanisms underlying joint diseases such as osteoarthritis (OA) and rheumatoid arthritis (RA). In a previous issue of Arthritis Research and Therapy, Berg and colleagues [1] have provided new insights in this field by showing for the first time that chondrocytes express chemerin and its cognate receptor, ChemR23, and that this system may be involved in cartilage degradation.

Chemerin is a secreted protein that exerts its functions by binding the G protein-coupled receptor ChemR23. It was first discovered as a chemotactic peptide directing macrophages and dendritic cells expressing ChemR23 toward sites of inflammation, being involved in both adaptive and innate immunity [2]. As often happens, regulatory proteins have pleiotropic functions. ChemR23 is also expressed by endothelial cells, where it is

*Correspondence: fiannone@reumbari.uniba.it

DiMIMP-Rheumatology Unit, School of Medicine, University of Bari, 70124 Bari, Italy up-regulated by pro-inflammatory cytokines, and chemerin strongly induces angiogenesis in vitro by promoting endothelial cell proliferation and remodeling through stimulation of matrix metalloproteinase (MMP) activity [3]. Interest in chemerin has grown since it was discovered in fat tissue as a novel adipokine secreted by adipocytes, which also express ChemR23. Chemerin is up-regulated in white fat cells upon IL-1 $\beta$ stimulation in vitro and chemerin serum levels are increased in obese patients; thus, chemerin may be the functional link between chronic inflammation and obesity, and obesityrelated disorders such as type 2 diabetes and cardiovascular diseases [4].

There is evidence that chemokines may play an important role in recruiting inflammatory cells into the joints and contribute to chronic synovitis in OA and RA [5]. Additionally, chemokines exert catabolic effects on cartilage. Functional chemokine receptors (CCR-1, CCR-2, CCR-3, CCR-5, CXCR-1, and CXCR-2) are detectable on human articular chondrocytes and up-regulated in OA cartilage. The interactions of these receptors with their ligands activate matrix degradation by inducing MMP-3 synthesis [6]. In their study, Berg and colleagues [1] explored whether chondrocytes also express ChemR23 and investigated the metabolic effects of chemerin stimulation on chondrocytes. A series of experiments was performed on human native cartilage or cultured chondrocytes isolated from joints of patients undergoing knee arthroplasty for severe OA, and patients subjected to autologous chondrocyte transplantation. Cartilage from young subjects undergoing reconstruction of anterior cruciate ligament was taken as normal control. ChemR23 and prochemerin transcripts were detected in chondrocyte cultures by reverse transcriptase PCR. Furthermore, ChemR23 and chemerin proteins were detected in chondrocytes in vitro by immunocytochemistry. These findings were confirmed by immunohistochemistry in cartilage biopsies, in which resident chondrocytes showed positive staining for both ChemR23 and chemerin. The effects of challenging isolated chondrocytes with recombinant chemerin in vitro were also investigated. Chemerin binding to ChemR23 led to 
increased phosphorylation of p44/42 mitogen-activated protein kinases (MAPKs) and Akt and blocking of MEK-1/2 signaling prevented phosphorylation of $\mathrm{p} 44 / 42$ MAPKs but not of Akt. This suggests that intracellular downstream events upon chemerin stimulation occur through the Akt/MEK/MAPK pathway. The most outstanding finding of this study was the demonstration that chemerin regulates the production of pro-inflammatory cytokines and MMPs by human chondrocytes in vitro. IL-1 $\beta$, TNF- $\alpha$, IL- 6 , and IL- 8 as well as MMP-13 and others MMPs were significantly increased in the supernatants of chondrocyte cultures stimulated with recombinant chemerin. It is noteworthy that OA chondrocytes secreted larger amounts of cytokines than chondrocytes from healthy subjects. MMPs play a key role in the remodeling of cartilage matrix and their increase is crucial in the induction of cartilage damage in $\mathrm{OA}$ and RA. In OA, MMP-13 has been shown to be the most important collagenase and, together with ADAMTS-5 (a disintegrin and metalloproteinase with thrombospondin motifs-5), causes the breakdown of the collagen and aggrecan framework of the extracellular matrix and initiates the destruction of cartilage [7]. Consistent with this study, chemerin production by chondrocytes has also been reported by Conde and colleagues [8]. They have shown that chondrocytes synthesize chemerin and its expression is increased by IL- $1 \beta$ stimulation. Furthermore, dexametasone increased the effect of IL- $1 \beta$ on mRNA expression of chemerin by chondrocytes suggesting that intra-articular injections of steroids as treatment of OA may damage the articular cartilage.

All together, these data strongly suggest that the chemerin/ChemR23 system is involved in cartilage damage in OA, and further studies will unravel its specific role. However, chemerin might not be one of the many redundant mediators that activate chondrocyte catabolic pathways, but may be a crucial link between obesity and OA. For years obesity has been regarded as a risk factor for developing OA in weight-bearing joints, but a growing body of evidence is showing that obesity is a mild inflammatory disease and that a pro-inflammatory network links fat tissue to obesity-related disease, including OA [9]. Chemerin is produced by adipocytes as well as chondrocytes and is up-regulated in both obesity and $\mathrm{OA}$, and might be a promising field of research to better understand and treat these two functionally associated diseases.

\section{Abbreviations}

IL, interleukin; MAPK, mitogen-activated protein kinase; MMP, matrix metalloproteinase; OA, osteoarthritis; RA, rheumatoid arthritis; TNF, tumor necrosis factor.

\section{Competing interests}

The authors declare that they have no competing interests

Published: 6 April 2011

\section{References}

1. Berg V, Sveinbjornsson B, Bendiksen S, Brox J, Meknas K, Figenschau Y: Human articular chondrocytes express ChemR23 and chemerin; ChemR23 promotes inflammatory signalling upon binding the ligand chemerin ${ }^{21-157}$. Arthritis Res Ther 2010, 12:R228.

2. Wittamer V, Franssen JD, Vulcano M, Mirjolet JF, Le Poul E, Migeotte I, Brézillon S, Tyldesley R, Blanpain C, Detheux M, Mantovani A, Sozzani S, Vassart G, Parmentier M, Communi D: Specific recruitment of antigen-presenting cells by chemerin, a novel processed ligand from human inflammatory fluids. J Exp Med 2003, 198:977-985.

3. Kaur J, Adya R, Tan BK, Chen J, Randeva HS: Identification of chemerin receptor (ChemR23) in human endothelial cells: chemerin-induced endothelial angiogenesis. Biochem Biophys Res Commun 2010, 391:1762-1768.

4. Ernst MC, Sinal CJ: Chemerin: at the crossroads of inflammation and obesity. Trends Endocrinol Metab 2010, 21:660-667.

5. Haringman JJ, Smeets TJ, Reinders-Blankert P, Tak PP: Chemokine and chemokine receptor expression in paired peripheral blood mononuclear cells and synovial tissue of patients with rheumatoid arthritis, osteoarthritis, and reactive arthritis. Ann Rheum Dis 2006, 65:294-300.

6. Borzi RM, Mazzetti I, Cattini L, Uguccioni M, Baggiolini M, Facchini A: Human chondrocytes express functional chemokine receptors and release matrixdegrading enzymes in response to C-X-C and C-C chemokines. Arthritis Rheum 2000, 43:1734-741.

7. lannone F, Lapadula G: The pathophysiology of osteoarthritis. Aging Clin Exp Res 2003, 15:364-372.

8. Conde J, Gomez R, Bianco G, Scotece M, Lear P, Dieguez C, Gomez-Reino J, Lago F, Gualillo O: Expanding the adipokine network in cartilage: identification and regulation of novel factors in human and murine chondrocytes. Ann Rheum Dis 2011, 70:551-559

9. lannone F, Lapadula G: Obesity and inflammation - targets for OA therapy. Curr Drug Targets 2010, 11:586-598.

doi:10.1186/ar3273

Cite this article as: lannone F, Lapadula G: Chemerin/ChemR23 pathway: a system beyond chemokines. Arthritis Research \& Therapy 2011, 13:104. 